\section{Recurrent origin of a sexually selected trait in Xiphophorus fishes inferred from a molecular phylogeny}

\author{
Axel Meyer*†, Jean M. Morrissey* \\ \& Manfred Schartl \\ * Department of Ecology and Evolution, and \\ + Program in Genetics, State University of New York, \\ Stony Brook, New York 11794-5245, USA \\ + Theodor-Boveri-Institut für Biowissenschaften. \\ Biozentrum der Universität Würzburg, Am Hubland, \\ 97074 Würzburg, Germany
}

DARwIN ${ }^{1}$ believed that sexual selection accounts for the evolution of exaggerated male ornaments, such as the sword-like caudal fin extensions of male fishes of the genus Xiphophorus, that appear detrimental to survival. Swordtails continue to feature prominently in empirical work and theories of sexual selection; the pre-existing bias hypothesis has been offered as an explanation for the evolution of swords in these fishes ${ }^{2,3}$. Based upon a largely morphological phylogeny, this hypothesis suggests that female preference to mate with sworded males arose in ancestrally swordless species, thus pre-dating the origin of the sword itself and directly driving its evolution. Here we present a molecular phylogeny (based on mitochondrial and nuclear DNA sequences) of Xiphophorus which differs from the traditional one: it indicates that the sword originated and was lost repeatedly. Our phylogeny suggests that the ancestor of the genus is more likely to have possessed a sword than not, thus questioning the applicability of the pre-existing bias hypothesis as an explanation for the evolution of this sexually selected trait.

The evolution of exaggerated male secondary sexual characteristics remains one of the most persistent problems in evolutionary biology, and many models of sexual selection have been offered to explain this phenomenon (reviewed in refs 4-7). Some of the most recent models are derived from the 'sensory drive' hypothesis (reviewed in ref. 8), which attempts to explain the origin and evolution of signal design and reception. In the context of sexual selection, the "sensory exploitation"7.9 11 and "preexisting bias ${ }^{\prime 2.3}$ hypotheses propose that the evolution of extreme male traits is driven by pre-existing female sensory biases that arose through physiological properties of female sensory systems. Thus, females evolve a sensory bias, which evolutionarily pre-dates and drives the subsequent origin and direction of the evolution of lavish male traits. Therefore, unlike other models of sexual selection, these hypotheses can be tested in a phylogenetic framework.

One of the two commonly cited (see for example refs 12,13) examples for the pre-existing bias hypothesis involves fishes of the genus Xiphophorus (for other examples, see ref. 14). Xiphophorus belong to the family Poeciliidae, freshwater fishes from Central America with internal fertilization accomplished by means of the gonopodium, the modified anal fin of males. Males of many species of this genus possess a ventrally elongated caudal fin, the sword. Various definitions of the sword have been used, yet experts on the fish generally regard any extension of the ventral caudal fin rays as a sword ( $R$. Borowsky, K. D. Kallman and $M$. Schartl, personal communication). Fishes of the genus Xiphophorus are commonly divided into swordtails and platyfishes, the latter being formerly placed into a different genus. Although most platyfishes do not possess swords, $X$. xiphidium and $X$. andersi exhibit small swords similar in length or even longer than those of some of the swordtail species ( $X$. pygmaeus, $X$. continens and $X$. birchmanni) (Fig. 1).

The present phylogenetic estimate for Xiphophorus (Fig. 1) is primarily based on morphological characters, many of which were derived from the gonopodium and its associated structures $^{15}$, but also uses allozyme variation for the northern swordtails ${ }^{16}$. In this phylogeny, the platyfishes are paraphyletic; the northern platyfishes are basal to the southern platies, which in turn are more closely related to the swordtails than to the other platies (Fig. 1). Recent phylogenetic work ${ }^{17}$, which adds behavioural characters, differs from the previous phylogeny in suggesting that a southern platy, $X$. maculatus, is the most basal member of the genus and that two sworded species, a platy ( $X$. xiphidium) and $X$. andersi, group with the swordtails rather than the platies.

Three criteria must be met to show that pre-existing biases have resulted in the evolution of swords Xiphophorus ${ }^{2}$. (1) Demonstration that female choice is based on variation in the trait. In choice experiments, female $X$. heller $i$ preferred to associate with males with longer swords over males with shorter swords $^{18}$. (2) Evidence must be provided that in species in which the males do not possess the trait, the females prefer to mate with males that do. In choice experiments, female platyfish ( $X$ maculatus) spent more time with conspecific males that had long, artificial swords attached (similar to those of $X$. helleri) over naturally swordless conspecific males ${ }^{2.3 .18}$. (3) The absence of the trait must be shown to be the primitive condition. This condition requires phylogenetic knowledge, and appears to be met, given the traditional phylogeny already described (Fig. 1). Because the platies are placed at the base of the genus and are generally swordless, it seems most parsimonious to assume that the common ancestor of the platyfishes and the more derived swordtails was likely to have been a swordless species. Hence, the preexisting bias hypothesis ${ }^{2.3}$ seems to explain the evolution of swords in Xiphophorus.

The applicability of the pre-existing bias hypothesis to Xiphophorus critically depends on the accuracy of the phylogeny. If the common ancestor of the genus was likely to have been sworded $^{16}$, this hypothesis would not hold because the female preference would not have pre-dated the origin of the sword in
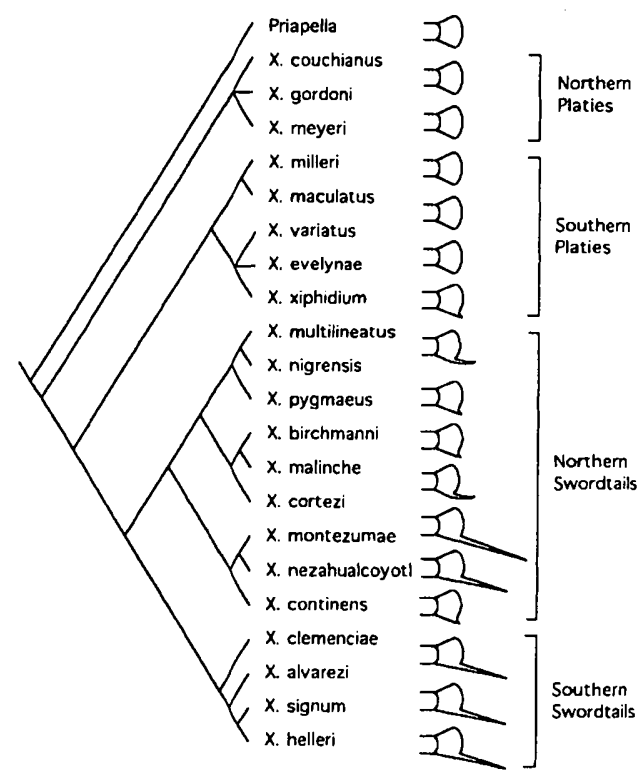

FIG. 1 Traditional morphology-based phylogenetic hypothesis for species of the genus Xiphophorus ${ }^{15.16}$ based on ref. 3 . The genus Xiphophorus has traditionally been divided into four groups (Fig. 1). The phylogenetic position of $X$. andersi has not been determined on the basis of morphological characters, and is therefore not included in this phylogeny. $X$. andersi has been placed as the sister group of the northern platyfishes. Priapalla is the sister group (its males do not have elongated tails) to Xiphophorus ${ }^{15,19.34}$. This phylogeny suggests that the swordless condition might be ancestral and the sworded condition is derived. 


\section{LETTERS TO NATURE}

Xiphophorus. In the traditional phylogeny, four of the seven synapomorphies that united swordtails were traits related to the sword $^{15}$. As the origin of the sword is of interest, one might want to avoid the danger of circularity and not include characters that are related to the sword in a phylogenetic analysis.

To provide an estimate of the phylogeny of Xiphophorus that is independent of the character to be studied, we constructed a DNA-based phylogeny. We included all 22 currently recognized species of the genus, and six outgroup species from the remaining three genera in the tribe Poeciliini ${ }^{\text {t9 }}$. The data set consists of 1,284 base pairs (bp) from two partial mitochondrial gene sequences (cytochrome $b$ and the control region) and one nuclear gene (the tyrosine kinase gene $X-s r c$ ). The data were analysed with the maximum parismony ${ }^{20}$ (Fig. 2) and neighbour-joining methods $^{21}$ (Fig. 3). Both methods yielded almost identical phylogenetic estimates which differ, however, from the previous phylogeny (Figs 1,2 and 3). The molecular and traditional phylogenies agree on the monophyly of the northern platies and of the northern swordtails, and on many relationships within these groups. But the molecular data suggest that the genus Xiphophorus is split not into the platyfishes and a derived group composed of northern and southern swordtails, but rather into two different groups: one consisting of the northern swordtails and the other of the southern swordtails plus the platyfishes. Within the latter group, the southern swordtails (except $X$. clemenciae, see later) appear to be the sister group of platies. Previously, all southern platies were believed to be a monophyletic group (Fig. 1); in our phylogeny they are paraphyletic, with Xiphophorus xiphidium as the sister species of northern platyfishes (Figs 2, 3).

$X$ iphophosus andersi ${ }^{22}$ has a number of morphological features aligning it to the southern swordtails (for example, it has a small sword and an elongated body) and some linking it to the southern platies (such as gonopodial structure and large head). Its intermediate molecular phylogenetic position as sister species to all platies would seem to confirm its midway morphology (Figs $2,3)$. Xiphophorus clemenciae, which is traditionally grouped with the southern swordtails (but see ref. 23) because of its close morphological resemblance, is nested among the southern platyfishes instead. Its mitochondrial DNA (mtDNA) does not closely match that of any extant species of Xiphophorus; further research will be necessary to test the possibility that this species originated by an ancient hybridization event ${ }^{24}$. Although hybridization in Xiphophorus has not been reported in nature, in labora-

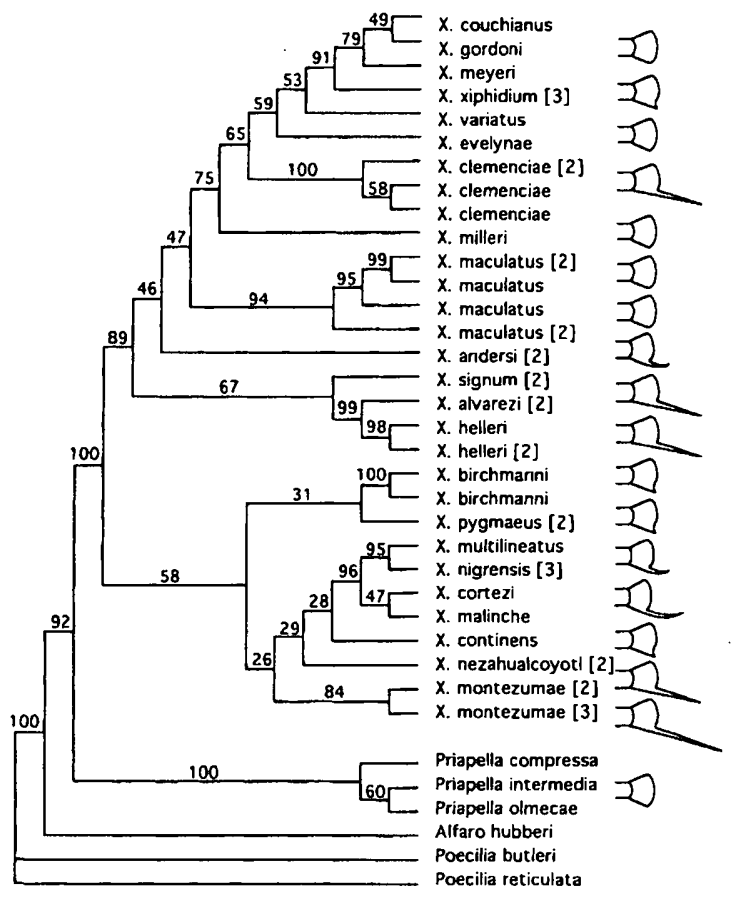

FIG. 2 Fifty per cent majority-rule maximum parsimony bootstrap tree (200 replications) and groupings compatible with it, based on the combined data set of $1,284 \mathrm{bp}^{35.20}$. We sequenced 52 partial control regions (402 bp), 47 partial cytochrome b genes ( $360 \mathrm{bp}$ ), and 42 partial $X$-src genes (522 bp). Sequences have been deposited in Genbank under accession numbers U06488-U06628. DNA sequences were determined after amplification by the polymerase chain reaction (PCR); details of the protocol and the primer sequences used for PCR amplification and direct sequencing have been published (except for the primer L15995 [L-Pro]: 5'-AACTCTCACCCCTAGCTCCCAAAG-3' for the control region, which was designed for this study) ${ }^{36-39}$. To improve computational feasibility of this large data set, individuals with sequences that were identical or differed in only one or two bp were combined, and IUPAC symbols were used in the variable sites (number of individuals pooled are given in parentheses after the species name). Thus, the number of taxa was reduced by 16 to 36 . In addition to all 22 species of Xiphophorus, the analysis included members from all other genera of the tribe Poeciliini ${ }^{19}$ : Priapella (intermedia, compressa, olmecae), Alfaro hubberi, and Poecilla (butleri and reticulata, the guppy), which were declared as the outgroup. Information on sampling localities and collectors for each specimen can be obtained from A.M. No additions or deletions were detected in cytochrome $b$, hence the alignment was unambiguous. The control region and $X$-src were aligned using CLUSTAL ${ }^{40}$, with adjustments by eye. $X$-src is a slowly evolving gene and no amino-acid substitutions were inferred among species of $X i p h o p h o r u s ;$ almost all variation detected was in the intron sequences. The alignments of $28 \mathrm{bp}$ of the control region and $26 \mathrm{bp}$ of $X$-src were questionable; these regions were excluded from the analyses. Data were analysed with maximum parsimony (MP) ${ }^{20}$ and neighbour-joining (NJ) methods ${ }^{21}$ (Fig. 3), and the robustness of the phylogenetic hypoth eses were tested by bootstrapping ${ }^{35}$. In all parsimony analyses, the following options were used: heuristic search, MULPARS option in effect, GAPMODE = MISSING, and TBR branch swapping. For the control region (no weights), only six shortest trees (length 335) $(\mathrm{Cl}=0.633, \mathrm{RC}=0.482$ ) were found. For cytochrome $b$ (no weights), 81 shortest trees (length 285 steps) $(\mathrm{Cl}=0.596, \mathrm{RC}=0.438$ ) were found. For $X$-src (no weights) 644 equally short trees were found (length 99$)(\mathrm{Cl}=0.778, \mathrm{RC}=0.664)$ were found. The phylogenetic information in the control region sequences seems most reliable because it resulted in the fewest shortest trees. In a regression analysis of all species of Xiphophorus, a 2:1 transition-to-transversion ratio (TS:TV) for the control region was observed. In cytochrome $b$, an 8:1 TS:TV was observed within the genus. In X-src, transitions and transversions were observed in approximately equal frequencies. In phylogenetic analyses of only $X$-src or cytochrome $b$ sequences, the bootstrap values tended to be low; they were much higher in analyses of the control region alone. Cytochrome $b$ provided little resolution at the $50 \%$ majority rule bootstrap level for deeper nodes, but strongly supported the monophyly of all species and groups of very closely related species. In some separate analyses of cytochrome $b$, the tree is rooted at the northern platies or $X$. andersi. Its topology tended to be susceptible to differential weighting of transitions and transversions. Also, $X$-src provided little resolution at the $50 \%$ bootstrap level, which was expected, given the few number of variable sites in this conservative gene. The tree shown is based on equal weights for transitions and transversions in the cytochrome $b$ and $X$ src genes, and transversions weighted twice transitions in the control region. The combined data set was robust to changes in weights for transversions in the different genes. Different weights caused only differences in the position of $X$. andersi (branching before or after the signum-alvarezi-helleri clade), the position of $X$. xiphidium in relation to the northern platies, the relation of cortezi and mallnche to the multilineatus-nigrensis clade, and the respective positions of continens and nezahualcoyotl. None of the changes due to different weighting regimes in the combined data set affected the reconstruction of the sword in the ancestor of the genus Xiphophorus (Fig. 4). If the topology of the traditional phylogenetic hypothesis is forced upon the total molecular data set, the number of evolutionary steps, or tree length, increases by over $10 \%$ ( 569 + versus 510 unweighted MacClade steps with soft polytomies) ${ }^{41}$. 


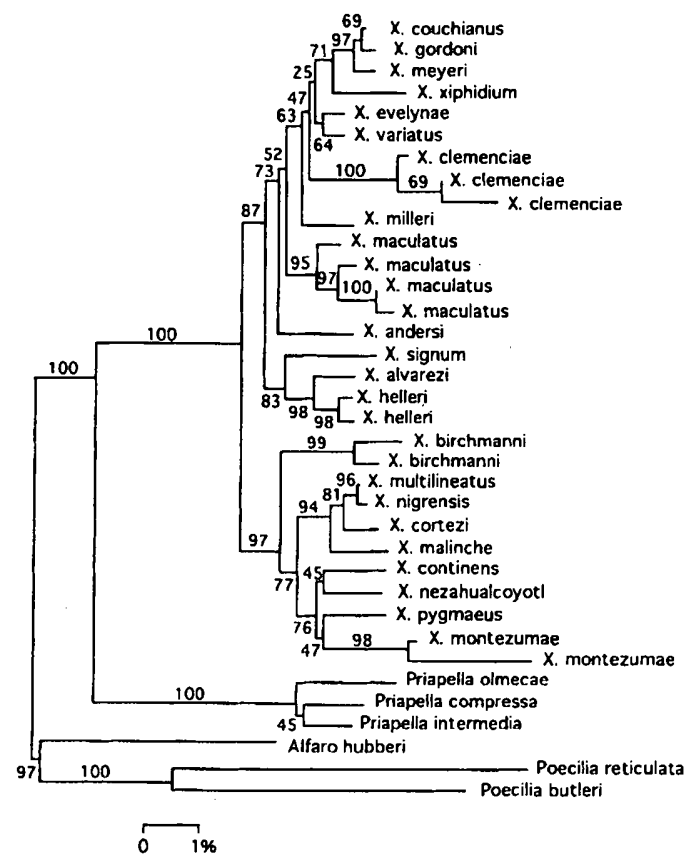

FIG. 3 Majority-rule bootstrap (500 replications) neighbour-joining trees $^{21}$ performed with the program MEGA ${ }^{42}$ on the distance matrix for the complete data set adjusted for multiple hits using Kimura's twoparameter model and pairwise deletion of gaps. Branches are drawn to scale, with the bar representing per cent divergence. The $\mathrm{NJ}$ analysis results in a topology that is almost identical to the MP phylogeny (Fig. 2), minor differences being the groupings of variatus and evelynae as sister species, and relationships among some members of the northern swordtail clade.

tory tests $X$. pygmaeus females prefer to mate with $X$. nigrensis males over conspecific males owing to their more elaborate courtship displays ${ }^{25,26}$.

In regards to the pre-existing bias theory for Xiphophorus, our phylogeny suggests that the ancestor for the entire genus possessed a sword (Fig. 4). Other evidence exists supporting the hypothesis of a sworded ancestor. Through hormone treatments, elongation of the caudal fin can be induced in several species of platyfish tested (for example, in $X$. milleri and $X$. maculatus) which normally do not express this trait ${ }^{27-29}$. These experiments demonstrate that 'sword' genes and the capability to express them during development are present in some platyfishes without swords. If the molecular phylogeny is correct, this capability might have been retained in these species from ancestrally sworded species (Figs 2, 3 and 4). Sword-like structures occur outside the genus Xiphophorus, for example in Poecilia petenensi. They are also present in low frequency in natural populations of the guppy, $P$. reticulata, and can be selected for artifically (J. A. Endler, personal communication). Swords might be phylogenetically old, and the capability to express swords might have been present even in the common ancester of all Xiphophorus s. $^{130}$ (Fig. 4).

It appears that swords were lost phylogenetically in some species of platies, despite a continued existence of a female preference for sworded males (in $X$. maculatus, for example ${ }^{2}$ ) and the capability of male platies to express caudal fin elongations after hormone treatment. The presence of a sword in males presumably confers a cost in terms of natural selection which might counteract any benefits due to sexual selection by increased mating success. Males with swords may suffer a decrease in swimming speed and an increase in visibility, resulting in an increased risk of predation. The potential costs in survivorship may have led to the loss of swords, despite a mating advantage of males with swords over males without them.

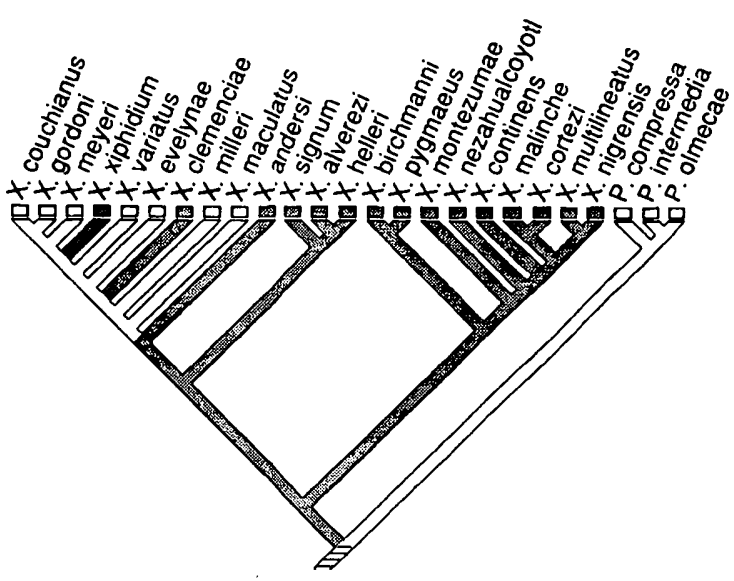

FIG. 4 Mapping of the traits 'no sword' and 'sword', and most parsimonious reconstruction of the ancestral states on the molecular phylogeny (Fig. 2) using MacClade ${ }^{41}$. The reconstruction of the evolution of the sword is based on the MP majority-rule bootstrap phylogeny; the minor differences between the MP and $\mathrm{NJ}$ analyses (Figs 2,3 ) have no effect on the reconstruction of the sword in the ancestor of the genus. As all species were found to be monophyletic, they are represented by only one terminal taxon. If the presence or absence of the sword is treated as a two-state character (sword-no sword), then the common ancestor most likely possessed a sword (4 steps). Forcing the ancestor of the genus to be swordless adds one more step to the evolution of the sword. Basolo ${ }^{3}$ defines several characters from the general character complex 'sword' and the sword itself ${ }^{2}$ as 'a coloured extension of the lower margin of the caudal fin'. Under her definition a sword is a coloured ventral extension $0.7-6.0$ times the length of the caudal fin, and a protrusion is a coloured ventral extension, 0.1 to 0.3 times the length of the caudal fin. If the characters are coded in this manner with regard to relative length ( $X$. birchmanni, $X$. pygmaeus, $X$. continens and $X$ $x$ iphidium have a protrusion) and treated as 'ordered' (that is, protrusion is an intermediate step in the evolution of swords from no swords, NS P.S, as suggested in ref. 41), then the common ancestor of all species of Xiphophorus most probably had a protrusion or a sword rather than no sword (9 steps). Under these conditions of reconstruction, 10 steps would be required if the ancestor was swordless. If the character evolution is assumed to be unordered (with three states), the common ancestor is likely to have had a sword (6 steps); forcing the ancestor to be swordless or to possess a protrusion adds a step to the character reconstruction (7 steps).

Female preferences and preferred male traits might be expected to be genetically linked ${ }^{31}$ and their positive genetic correlation has been demonstrated ${ }^{32,33}$. The evolutionary dissociation of swords and female preference therefore seems odd. Based on the molecular phylogeny, it appears as if caudal fin extensions can be lost and regained (for example, in $X$. xiphidium) (Fig. 4). Basolo's ${ }^{2}$ finding of a preference for swords in a species whose males are lacking swords is intriguing; but in light of the new phylogenetic hypothesis, the puzzle may not be why males in most species of platyfish never evolved swords despite a female preference for them, but rather why swords were lost in the presence of an apparently retained bias in females to mate with males with swords.

Received 17 November 1993; accepted 17 January 1994.

1. Darwin, C. The Descent of Man, and Selection in Relation to Sex (Murray, London, 1871).

2. Basolo, A. L. Science 250, 808-810 (1990).

3. Basolo, A. L. Sclence 283, 1426-1427 (1991).

. Bradbury. J. W. \& Anderson. M. B. (eds) Sexual Selection: Testing the Atternatives (Wiley, Chichester, UK, 1987).

5. Maynard Smith, J. in Sexual Selection: Testing the Altematives (eds Bradbury, J. W. \& Anderson, M. B.) 9-20 (Wiley, Chichester, UK, 1987).

6. Maynard Smith. J. Trends Ecol. Evol. 6, 146-151 (1991)

7. Kirkpatrick, M. \& Ryan, M. J. Nature 350, 33-38 (1991)

8. Endler, J. A. Am. Nat. 139, S125-S153 (1992).

9. Kirkpatrick. M. in Sexual Selection: Testing the Alternatives (eds Bradbury, J. W. \& Anderson. M. B.) 67-82 (Wiley, Chichester, UK, 1987).

10. Ryan, M. Oxf. Surv. Evol. Biol. 7, 157-195 (1990). 


\section{LETTERS TO NATURE}

11. Ryan, M. Jox J H. Wilcrynski, W. \& Rand, A. S. Nature 343, 66-68 (1990).

12. Alcock, Animal Behavior 5 th edn (Sinaver, Su s. Nater 1993)

13. Krebs, A \& Davies. Oxford, 1993)

14. Ryan, M. \& Rand, A S. Phil Trans, R. Soc B 340, 187-195 (1993)

15. Rosen, D. E. Bull. Am. Mus. nat. His. 182, 268-375 (1979).

16. Rauchenberger, M., Kallman, K. O. \& Morizot, D. C. Am. Mus. nat. His. Nov. 2976, 1-41 (1990).

17. Haas, V. in Trends in lchthyology (eds Schrōder, J. H., Bauer, J. \& Schartl, M.) 279-288 (Blackwell, London, 1993).

18. Basolo. A. L. Anlm. Behav. 40, 332-338 (1990)

19. Rosen, D. E. \& Bailey, R. M. Bull. Am. Mus, nat. Hist. 126, 1-176 (1963).

20. Swofford, D. L. Phylogenetic Analysis Using Parsimony Version 3.1.1 (Illinois Natural Histon Survey, Champaign, 1993)

21. Saitou, N. \& Nei, M. Molec. Biol. Evol. 4, 406-425 (1987)

22. Meyer, M. K. \& Schartl, M. Senkenbergiana biol, 60, 147-151 (1980)

23. Morizot, D. C. \& Siciliano, M. J. Genetics 102, 539-556 (1982).

24. Dowling, T. E. \& DeMarais, 8. D. Nature 362, 444-446 (1993).

25. Ryan, M. J. \& Wagner, W. E. Jr Science 236, 595-597 (1987)

26. Partridge, L \& Harvey, P. H. Nature 328, 377 (1987).

27. Gordon, M., Cohen, H. \& Nigrelli, R. F. Am. Nat. 77, 569-572 (1943).

28. Drwillo M. Verh Ot Zool Ges 1982 152-159 (1963)

29. Zander, C. D. \& Dzwillo, M. Z. Wissen. Zool. 178, 276-315 (1969).

30. Winquist, S. T. et at. Science 253, $1426(1991)$.

31. Fisher. R. A. The General Theory of Natural Selection 2nd edn (Dover, New York, 1958).

32. Houde, A. \& Endler, J. A. Sclence 248, 1405-1408 (1990).

33. Bakker, T. C. M. Nature 363, 255-257 (1993).

34. Rosen, D. E. \& Kallman, K. D. Am Mus. Hat. His. Nov. 2379, 1-29 (1969)

35. Felsenstein, J. Evolution 39, 783-791 (1985).

36. Kocher, T. D. et al. Proc. natn. Acad. Sci. U.S.A. 86, 6196-6200 (1989)

37. Meyer, A., Kocher, T. D., Basasibwaki, P. \& Wilson, A. C. Nature 347, 550-553 (1990)

38. Meyer, A. \& Lydeard, C. Proc. R. Soc. Lond. 8 254, 153-162 (1993)

39. Robertson, S. M thesis, Ludwigs Maximillians Univ, München (1989).

40. Higgins, D. G. \& Sharp, P. M. CABIOS S, 151-153 (1989)

41. Maddison, W. P. \& Maddison, D. R. Macclade Ver. 3.0. Analysis of Phylogeny and Character Evolution (Sinauer, Sundertand, 1992).

42. Kumar, S., Tamura, K. \& Nei, M. MEGA Molecular Evolutionary Genetics Analysis Vers. 1.0 (Inst. of Mol. Evol. Genetics, Pennsyivania State Univ. 1993)

43. Meyer, M. K. Cour. Forsch.Inst. Senckenberg 94, 123-130 \{1987\}.

ACKNOWLEDGEMENTS. We thank R. Borowsky, J. Endler, R. McKeand and particularly $K$. Kallmann for specimens: $S$. Robertson for $X$-srC primer design; C. Davis for technical assistance and A. Basolo, R. Borowsky, J. Endler, K. Kallmann. C. Lydeard, E. Mayr, E. Prager and M. Ryan for comments on the manuscript. Part of this work was completed while A.M. was an Alfred $P$. Sloan Postdoctoral Fellow in the laboratory of the late Allan C. Wilson at the University of California at Berkeley. A.M. is supported by the US NSF: M.S. is supponed by the DFG, Germany.

\section{The generic viewpoint assumption in a framework for visual perception}

\section{William T. Freeman}

Mitsubishi Electric Research Laboratories, 201 Broadway, Cambridge, Massachusetts 02139, USA

A visual, system makes assumptions in order to interpret visual data. The assumption of 'generic view'1-4 states that the observer is not in a special position relative to the scene. Researchers commonly use a binary decision of generic or accidental view to disqualify scene interpretations that assume accidental viewpoints ${ }^{5}{ }^{10}$. Here we show how to use the generic view assumption, and others like it, to quantify the likelihood of a view, adding a new term to the probability of a given image interpretation. The resulting framework better models the visual world and reduces the reliance on other prior assumptions. It may lead to computer vision algorithms of greater power and accuracy, or to better models of human vision. We show applications to the problems of inferring shape, surface reflectance properties, and motion from images.

Consider the image of Fig. la. Perceptually, there are two possible interpretations: a bump, lit from the left, or a dimple, lit from the right. Yet many shapes and lighting directions (Fig. $1 b$ ) could explain the image. How should a visual system choose?

We note that the ridges in shapes 2-4 of Fig. $1 b$ must line up with the assumed light direction. We can study the accidentalness' of this alignment by exploring how the image of the illuminated shape changes as we perturb the azimuthal light direction. Figure $1 c$ shows that shape 3 presents images similar to that in

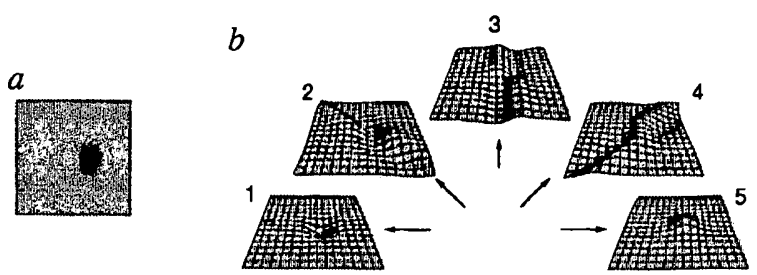

shapes for different assumed light directions

C shape 3
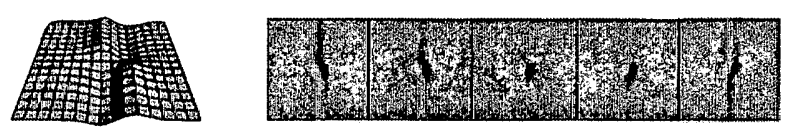

assumed
light direction
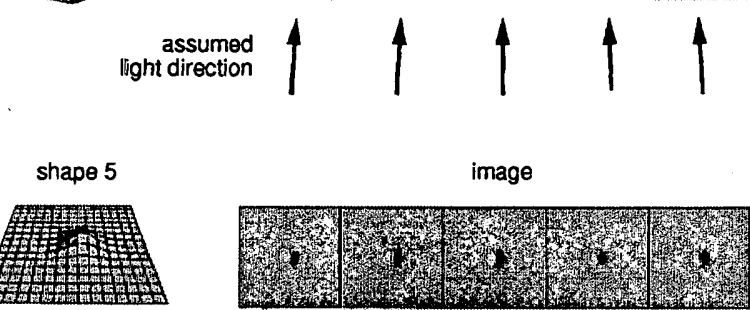

assumed

light direction
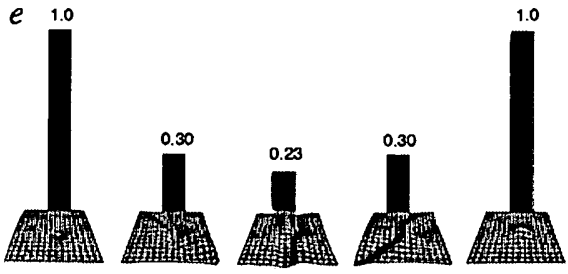

FIG. 1 a, Perceptually, this image has two possible interpretations. It could be a bump, lit from the left, or a dimple, lit from the right. $b$, Mathematically, there are many possibilities. The five shown here were found by a linear shape from shading algorithm, assuming shallow incident light from different azimuthal directions and the boundary conditions described in ref. 8 . Shapes $2-4$ require coincidental alignment with the assumed light direction. For shape 3 in $c$, the rendered image changes quickly with assumed light angle; only a small range of light angles yields an image like that shown in a. The generic view term of the scene probability equation, equation (7), penalizes an interpretation that has high image derivatives with respect to the generic variable, in this case light direction. For shape 5 in $d$, a much larger range of light angles gives the observed image. If all light directions are equally likely, shape 5 should be the preferred explanation. The probabilities of the candidate shapes, found using equation (7), are shown in e. The results favour shapes 1 and 5 , in agreement with the perceptual appearance of a.

Fig. I $a$ only for a small range of assumed light directions. The bump in Fig. 1d (shape 5) presents images like that in Fig. 1a over a broader range of light directions. If all azimuthal light directions are equally likely, shape 5 has more chances to create the image in Fig. 1a than does shape 3.

To quantify such probabilities, we use a bayesian framework (as in ref. 11, for example). This combines the data (Fig. 1a) with known or estimated prior probabilities to find the posterior probability of each candidate shape.

We treat the azimuthal light direction as a random variable an example of what we call a generic variable, $\bar{x}$, with prior probability density $P_{\dot{x}}(\vec{x})$. (We use subscripts to distinguish 